\title{
miR-141 promotes colon cancer cell proliferation by inhibiting MAP2K4
}

\author{
LEI DING, LI-LI YU, NING HAN and BU-TIAN ZHANG \\ Department of Radiology, The Third Hospital of Jilin University, Changchun, Jilin 130000, P.R. China
}

Received April 30, 2015; Accepted September 9, 2016

DOI: $10.3892 / 01.2017 .5653$

\begin{abstract}
MicroRNAs (miRNAs or miRs) can function as tumor-suppressor or oncogenic genes. Upregulation of miRNA-141 has been frequently observed in colorectal cancer (CRC) samples. The experimentally observed targets of miR-141 include the tumor-suppressor gene mitogen-activated protein kinase kinase 4 (MAP2K4). The aim of the present study was to investigate the role of miR-141 in the proliferation of colonic cancer. Western blotting, immunohistochemistry and reverse transcription-quantitative polymerase chain reaction were used to detect the expression levels of miR-141 and MAP2K4 in colonic adenocarcinoma (CAC) and adjacent non-cancerous (NC) tissue samples, as well as in human CAC cell lines (HT29, T94 and LS174). MTT assay was used to investigate the proliferation and apoptosis of these three cell lines. The expression levels of miR-141 were significantly upregulated in clinical samples of CAC, compared with adjacent NC tissues. By contrast, MAP2K4 was downregulated in $\mathrm{CAC}$. The in vitro assays demonstrated that overexpression of miR-141 resulted in cell proliferation of CAC by inhibiting MAP2K4 activity. Our study suggests that targeting the miR-141-MAP2K4 signaling pathway may represent a novel approach for the treatment of CRC.
\end{abstract}

\section{Introduction}

Colorectal cancer (CRC) is one of the most commonly diagnosed cancers, and is a common cause of cancer-associated mortalities in developed countries $(1,2)$. In China, CRC is the fifth most common cancer type and the fourth most common cause of cancer-associated mortality (3). CRC prognosis is dependent on tumor stage at presentation (4). The 5-year overall survival ranges from $93 \%$ for stage I patients to $8 \%$ for stage IV patients (5). Currently, the only curative treatment

Correspondence to: Dr Li-Li Yu, Department of Radiology, The Third Hospital of Jilin University, 126 Xiantai Street, Changchun, Jilin 130000, P.R. China

E-mail: ct_yl1@126.com

Key words: miR-141, mitogen-activated protein kinase kinase 4, colon cancer, apoptosis is surgical resection, while a modest number of patients that survive benefit from chemotherapy (6). Despite numerous efforts having been made in the past few decades, CRC survival rates have not been significantly improved (7). As a result, novel biomarkers that are of clinical value are thus urgently required to facilitate early detection and allow personalized treatment strategies for patients at high risk of CRC, thus improving the compliance rates (8). A family of small regulatory RNAs termed microRNAs (miRNAs or miRs) has emerged with important functions in development, cell differentiation, and regulation of cell cycle and apoptosis (9-11).

miRNAs are a class of short 22 nucleotide-long non-coding RNAs that repress protein translation through binding to their target messenger RNAs (mRNAs) $(12,13)$. Bioinformatics and cloning studies have estimated that miRNAs may regulate $30 \%$ of all human genes and control hundreds of gene targets (14). In the past decade, a particular important role by miRNAs in tumor pathogenesis has surfaced (15). Indeed, globally abnormal miRNA expression patterns can classify human cancers, invasion, progression and response to therapy (16). It has been well-documented that certain specific miRNAs contribute to cell transformation, proliferation and tumorigenesis by serving as targets of genomic lesions that are associated with activation of oncogenes and inactivation of tumor suppressor genes in cancer cells, including amplification, deletion and epigenetic silencing (17). In addition, miRNAs can provide functional links downstream of classic oncogenic and tumor-suppressor signaling pathways (18). Furthermore, numerous studies have demonstrated the proand anti-tumorigenic activities of specific miRNAs both in vitro and in vivo $(19,20)$.

miRNA expression profiling in stage III CRC identified $>11$ miRNAs, including miR-21 and miR-141, that were significantly upregulated (21). It has been well documented that miR-21 functions as an oncogene and modulates tumorigenesis through the regulation of genes such as B-cell lymphoma-2 (22). miR-141 has been also implicated in tumorigenesis of several types of cancer, including nasopharyngeal cancer (23). An early study revealed that upregulation of miR-141, which was closely associated with late stage and poor survival in CRC, was only observed in CRC patients' blood, but not in cancer tissues, suggesting that such an increase may be derived from inflammatory responses in these patients (1). However, a more recent study demonstrated that miR-141 was significantly elevated in CRC tissue samples at stage III, compared 
with non-cancerous (NC) adjacent colorectal mucosa (21). Experimentally validated target genes of miR-141 include mitogen-activated protein kinase kinase 4 (MAP2K4) (23). MAP2K4 is a tumor suppressor gene that can phosphorylate c-Jun N-terminal kinase or p38 with dual specificity, resulting in the activation of the stress-activated protein kinase pathway, which has been associated with apoptosis and neoplastic transformation $(24,25)$.

In the present study, the hypothesis that miR-141 promotes cell proliferation of colon cancer by inhibiting MAP2K4 was tested. The results demonstrated that miR-141 was significantly upregulated in CRC cancer tissues, but barely detected in NC adjacent colorectal mucosa. By contrast, the levels of MAP2K were significantly elevated in normal tissues, but it was present at less detectable levels in CRC. In addition, it was further functionally confirmed that miR-141 inhibited MAP2K4 in a variety of colon cancer lines, thus promoting the proliferation of cancer cells. Our results suggest that miR-141 can be a therapeutic target for the treatment of CRC.

\section{Materials and methods}

Cell culture. The human colon carcinoma cell lines HT29, T84 and LS174 (ATCC, Manassas, VA, USA) were maintained in RPMI-1640 medium supplemented with $10 \%$ fetal bovine serum (ATCC). The cells were grown in a humidified incubator at $37^{\circ} \mathrm{C}$ with $5 \% \mathrm{CO}_{2}$.

Bioinformatics analysis. To investigate whether miR-141 targets MAP2K4, a bioinformatics analysis was performed using TargetScan (www.targetscan.org) and microRNA.org (http://www.microrna.org/microrna/home.do). miR-141 was shown to target sites 75-81 and 192-199 of the 3'-untranslated region (UTR) of MAP2K4 mRNA.

Reverse transcription-quantitative polymerase chain reaction $(R T-q P C R)$. Total RNAs were extracted with TRIzol (Invitrogen; Thermo Fisher Scientific, Inc., Waltham, MA, USA). Dried RNA pellets were re-suspended in appropriate volumes of diethylpyrocarbonate-trated $\mathrm{H}_{2} \mathrm{O}$ (Sigma-Aldrich; Merck Millipore, Darmstadt, Germany). Complementary DNA synthesis was achieved with miR-141-specific, U6 small nuclear RNA (snRNA)-specific or oligo-dT primers using SuperScript II Reverse Transcriptase (Invitrogen; Thermo Fisher Scientific, Inc.). qPCR was performed using an ABI 7300 Real-Time PCR System (Applied Biosystems; Thermo Fisher Scientific, Inc.). The cycling conditions were as follows: $95^{\circ} \mathrm{C}$ for $1 \mathrm{~min}$, followed by 40 cycles at $55^{\circ} \mathrm{C}$ for $30 \mathrm{sec}$ and $70^{\circ} \mathrm{C}$ for $30 \mathrm{sec}$. miR-141 or MAP2K4 mRNA levels were determined relative to U6 or GAPDH expression, respectively, using a SYBR Green PCR kit (Qiagen $\mathrm{GmbH}$, Hilden, Germany). Fold-change in expression was determined by the quantification cycle $(\mathrm{Cq})$ method using the formula $2^{-\Delta \Delta \mathrm{Cq}}(26)$. The primers used were as follows: U6 snRNA forward, 5'-TGCGGGTGCTCGCTTCGGCAGC-3' and reverse, 5'-GGGTCCGAGGTGCACTGGATACGACAA AATATGG-3'; miR-141 forward, 5'-ATCGCCAGGATAAAT TGACGCA-3' and reverse, 5'-CCGCCTTAACACTGTCTG GTA-3'; MAP2K4 forward, 5'-GATGAATCCAAAAGGCCA
AA-3' and reverse, 5'-TCAATCGACATACATGGGAGAG-3'; and GAPDH forward, 5'-CTCCCGCTTCGCTCTCTG-3' and reverse, 5'-CTGGCGACGCAAAAGAAG-3'.

Western blotting. Whole cell lysates were re-suspended in $1 \mathrm{X}$ SDS loading buffer, boiled at $95^{\circ} \mathrm{C}$ for $5 \mathrm{~min}$ and centrifuged AT $3,000 \times g$ for $5 \mathrm{~min}$ at $4^{\circ} \mathrm{C}$. Supernatants were resolved on $10 \%$ SDS-PAGE and transferred onto polyvinylidene difluoride membranes (Bio-Rad Laboratories, Inc., Hercules, CA, USA). Membranes were blocked in 5\% nonfat milk in PBS with Tween-20 (PBST) [10 mM phosphate buffer ( $\mathrm{pH} 7.2)$, $150 \mathrm{mM} \mathrm{NaCl}$ and $0.1 \%$ Tween-20] for $60 \mathrm{~min}$, washed three times with PBST and incubated with anti-MAP2K4 antibody (1:500; cat. no. sc-376838; Santa Cruz Biotechnology, Inc., Dallas, TX, USA) at $4^{\circ} \mathrm{C}$ overnight. The blots were probed with anti- $\beta$-actin antibody (1:500; cat. no. A-5316; Sigma-Aldrich; Merck Millipore) as a loading control. Membranes were washed three times with PBST, incubated with horseradish peroxidase (HRP)-conjugated secondary antibody (1:5,000; cat. no. 115-035-003; Jackson ImmumoResearch, West Grove, PA, USA) and developed with Immun-Star HRP Substrate (Bio-Rad Laboratories, Inc.).

Immunohistochemistry. Briefly, the tissue samples were fixed in buffered formalin for $24 \mathrm{~h}$, dehydrated in $70 \%$ ethanol, paraffin-embedded and sectioned $(5-\mu \mathrm{m})$. The slides were incubated with proteinase K (Roche Diagnostics, Indianapolis, IN, USA) for $20 \mathrm{~min}$ to help uncover the hidden antigens. Following incubation with the primary antibody against MAP2K4 at 1:100 dilution overnight at $4^{\circ} \mathrm{C}$, the sections were washed with TBS containing Tween-20 (TBST) prior to being incubated with the HRP-conjugated secondary antibody diluted in TBS. Sections were washed again with TBST, and the secondary antibody was detected using the VECTASTAIN $^{\circledR}$ Elite ABC HRP kit (Thermo Fisher Scientific, Inc.) for $30 \mathrm{~min}$. Two independent pathologists evaluated the intensity of cytoplasmic staining of MAP2K4 on a scale of 0 to 4 ( 0 corresponds to the absence of staining and 4 is the highest degree of staining). The range of the scale is 2-3 for $\mathrm{NC}$ and 0-1 for colonic adenocarcinoma (CAC).

Cell proliferation assay. MTT assay was performed to estimate the effect of miR-141 on human CAC cell proliferation, as previously described (27). Cells were seeded into 96-well plates (5,000 cells/well in $200 \mu \mathrm{l}$ medium) and incubated for $24 \mathrm{~h}$ at $37^{\circ} \mathrm{C}, 5 \% \mathrm{CO}_{2}$. Pancreatic ductal adenocarcinoma cells were transfected with miR-141 mimics (miR-141) or miR-141 antagomirs (Ant-mIR-141) (IDT DNA, Coralville, IA, USA) using Lipofectamine 2000 (Thermo Fisher Scientific, Inc.). Cells cultured in complete medium were used as a blank control. At the end of culturing, $20 \mu \mathrm{l}$ of a $5 \mathrm{mg} / \mathrm{ml}$ MTT solution (Sigma-Aldrich; Merck Millipore) was added per well, and the cells were incubated for another $4 \mathrm{~h}$ at $37^{\circ} \mathrm{C}$. Supernatants were then removed, and formazan crystals were dissolved in $150 \mu 1$ dimethyl sulfoxide (Sigma-Aldrich; Merck Millipore). Finally, the optical density was determined at $490 \mathrm{~nm}$ using Victor 3 Multi-Label Microplate Reader (PerkinElmer, Inc., Waltham, MA, USA). In each assay, five parallel wells were analyzed, and the results were collected as the mean of $\geq 3$ independent experiments. 


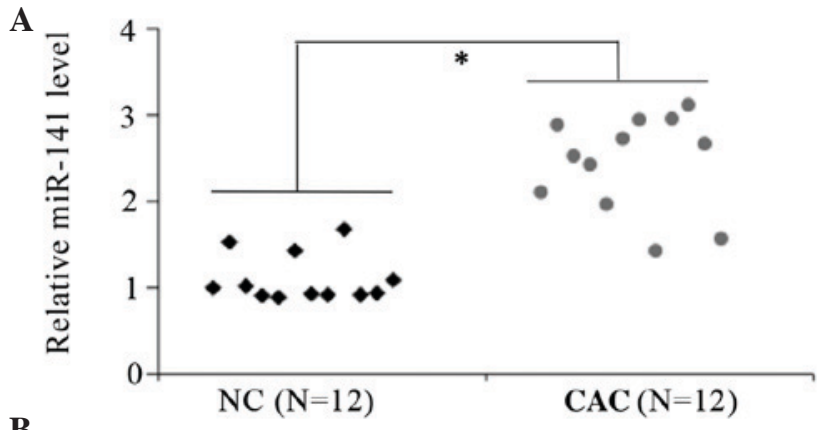

B

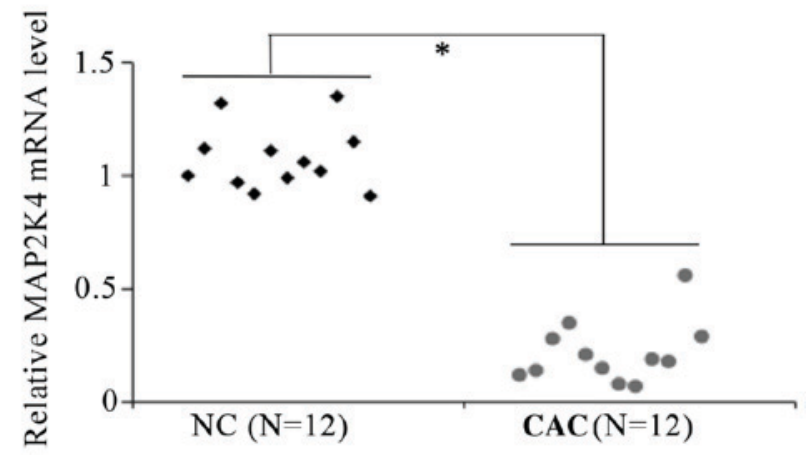

C

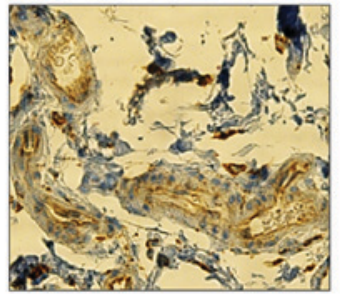

$\mathrm{NC}$

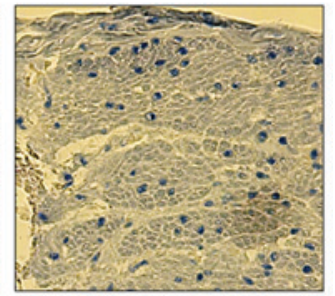

CAC
Figure 1. Expression of miR-141 and MAP2K4 in colon cancer specimens. The expression levels of (A) miR-141 or (B) MAP2K4 were determined by reverse transcription-quantitative polymerase chain reaction in both $\mathrm{CAC}$ and NC adjacent tissues. ${ }^{*} \mathrm{P}<0.01(\mathrm{n}=12)$. (C) Representative immunohistochemical staining of MAP2K4 performed on 12 specimens of CAC and 12 of $\mathrm{NC}$ tissues (magnification, $\mathrm{x} 200$ ). CAC, colonic adenocarcinoma; $\mathrm{NC}$, and non-cancerous; miR, miRNA; MAP2K4, mitogen-activated protein kinase kinase 4; mRNA, messenger RNA.

Luciferase assay. cDNA strands corresponding to the 3'-UTR of MAP2K4 mRNA containing the wild-type (WT) or mutant seed sequence (the distal one) of miR-141 were synthesized and the complementary strands were annealed at $75^{\circ} \mathrm{C}$ for $2 \mathrm{~min}$, cooled to room temperature for $5 \mathrm{~min}$ and cloned into the pGL4.32 vector (Promega Corporation, Madison, WI, USA) immediately downstream of the firefly luciferase (FL) gene. The resulting plasmids were sequence-validated prior to further analysis. HT29 cells were transfected with the plasmid for $48 \mathrm{~h}$ using Lipofectamine 2000, and luciferase assays were performed using the luciferase assay system (Promega Corporation) and the Victor $3 \mathrm{~V}$ plate reader (PerkinElmer, Inc., Waltham, MA, USA), according to the manufacturer's protocol.

Statistical analysis. Data are expressed as the mean \pm standard deviation. Student's $t$-test was used to compare the differences between two groups. Statistical analyses were performed using Microsoft Excel 2013 (Microsoft Corporation, Redmond, WA,
USA). $\mathrm{P}<0.05$ was considered to indicate a statistically significant difference.

\section{Results}

miR-141 is upregulated, but MAP2K4 is downregulated, in $C R C$. The levels of miR-141 and MAP2K4 were determined in CRC tissue specimens. Total RNAs were extracted from a total of 12 colon cancer and NC adjacent tissue samples for assessment of miR-141 and MAP2K4 mRNAs levels by qPCR. The same tissues were formaldehyde-fixed, paraffin-embedded and subjected to immunohistochemical staining. It was observed that miR-14 was significantly upregulated in CRC cancer tissues (Fig. 1A). By contrast, MAP2K4 mRNA levels decreased by an average of $>60 \%$ (Fig. 1B), compared with the $\mathrm{NC}$ adjacent colon mucosa. In addition, positive cytoplasmic staining for MAP2K4 was observed in the normal colon, but not in cancer tissues (Fig. 1C), with the use of immunohistochemistry. These results suggest that MAP2K4 is a target of miR-141 in CRC.

miR-141 promotes cell proliferation in vitro. To investigate the effect of miR-141 on CRC cancer cell growth, human CAC cell lines, including HT29, T84 and LS174, were transiently transfected with miR-141 mimics or miR-141 antagomirs (Ant-miR-141) and their respective non-specific controls. The RT-qPCR analysis revealed that the levels of miR-141 increased by 3-4-fold with transfection of miR-141 mimics in all three CAC cell lines (Fig. 2A). By contrast, transfection of miR-141 antagomirs led to a significant decrease in miR-141 levels (Fig. 2B), consistent with a previous finding (28).

The MTT proliferation assay was performed in cancer cell lines. The results indicated that miR-141 mimics promoted, while miR-141 antagomirs inhibited, cancer cell growth $(\mathrm{P}<0.01)$ in all three CAC cell lines (Fig. 2C-E). The cell cycle distribution was also analyzed in HT29 cells, and it was noticed that a larger proportion of cells transfected with miR-141 antagomirs accumulated in the G1 phase, whereas the $\mathrm{S}$-phase population significantly decreased $(\mathrm{P}<0.01$; data not shown). These results suggested that miR-141 acts as an oncogene to promote cancer cell proliferation in the pathogenesis of CRC.

MAP2K4 constitutes a direct target of miR-141 in CRC. MAP2K4 is a putative tumor-suppressor gene and an experimentally observed target gene for miR-141 (25). To investigate whether miR-141 targets MAP2K4 in CRC, a bioinformatics analysis was performed with the use of TargetScan and miRanda, and multiple miR-141 target sites in MAP2K4 were identified. Next, it was validated whether miR-141 directly targets MAP2K4 mRNA in CAC cells. An increase in intracellular concentration of miR-141 inhibited, while elevated miR-141 antagomirs remarkably increased, the protein levels of MAP2K4 in all three human CAC cell lines (Fig. 3A).

In order to further confirm the direct interaction between miR-141 and MAP2K4, a miR-141 target site was introduced at the 3'-UTR of MAP2K4 mRNA, downstream of the FL reporter gene, the regulation of which could be mediated by miR-141. In addition, a mutated version of this target site was used as a negative control. HT29 cells were transfected with 
A

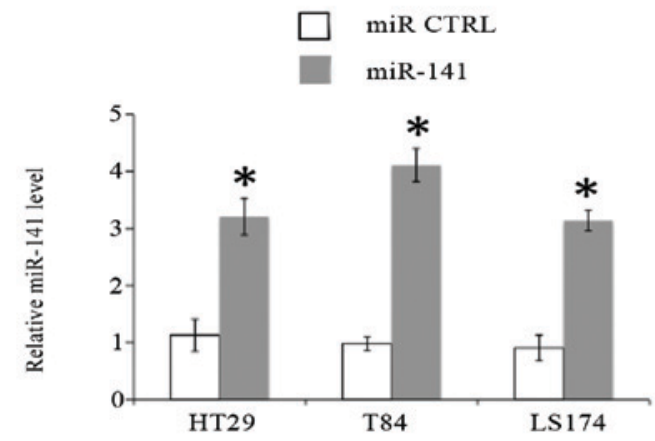

C

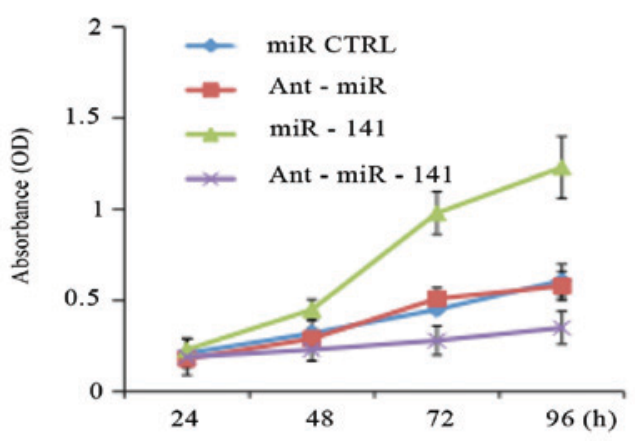

B

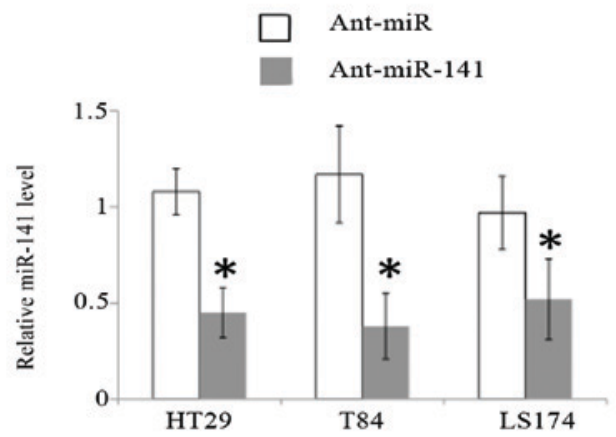

D

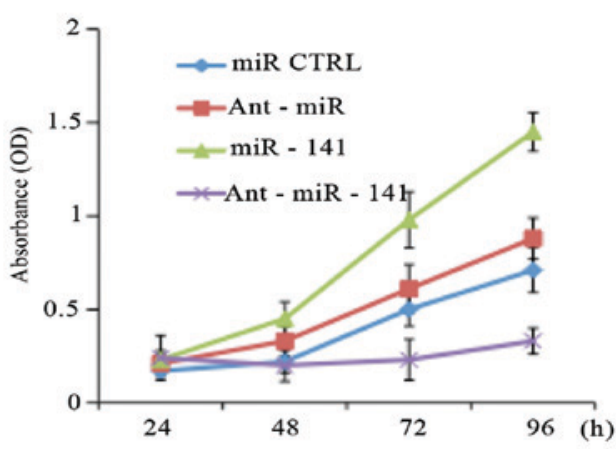

$\mathbf{E}$

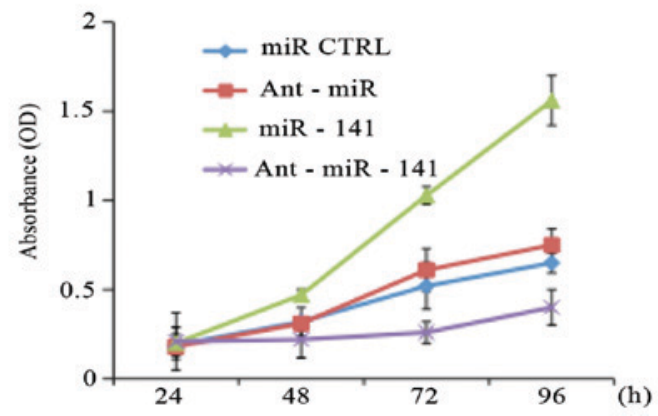

Figure 2. miR-141 promotes the proliferation of CAC cells in vitro. (A and B) The expression levels of miR-141 were determined at $48 \mathrm{~h}$ post-transfection in CAC cell lines (HT29, T84 and or LS174) transfected with (A) miR-141 mimics or (B) Ant-miR-141 and their respective controls (miR CTRL or Ant-miR, respectively; $40 \mathrm{nM}$ ) by reverse transcription-quantitative polymerase chain reaction. The proliferation of CAC cell lines (C) HT29, (D) T84 and (E) LS174, which were transiently transfected with miR-141 mimics, Ant-miR-141 or their respective controls, was analyzed by MTT proliferation assay. "P<0.01 (n=3). miR, microRNA; CTRL, control; Ant, antagomir; OD, optical density.; CAC, colonic adenocarcinoma.

the control FL plasmid, the FL plasmid with the miR-141 target site, or the FL plasmid with a mutation of the miR-141 target site. In addition, the Renilla luciferase reporter plasmid was co-transfected as an internal reference. As shown in Fig. 3B, a significant decrease in FL activity was observed in cells transfected with the FL reporter plasmid with WT miR-141 target site. By contrast, a de-repression in FL activity was observed in cells transfected with miR-141-mutant FL reporter plasmid.

Taken together, these results indicate that MAP2K4 is a direct target of miR-141 in CRC cells.

miR-141 supports CRC cell growth by inhibiting MAP2K4. To elucidate whether the growth-promoting effect of miR-141 is mediated by the repression of MAP2K4 in CRC cells, HT29 cells were transfected with small interfering RNA against MAP2K4. Then, cell growth was examined by MTT assay. As shown in Fig. 4, the MTT assay results demonstrated that gene silencing of MAP2K4 led to the proliferation of HT29 cells. Furthermore, the inhibitory effects of miR-141 antagomirs on cell growth were reversed when MAP2K4 was downregulated.

Collectively, these results suggest that miR-141 is involved in the proliferation of CRC cells by directly inhibiting MAP2K4.

\section{Discussion}

A comprehensive detailing of the molecular mechanisms underlying CRC initiation and progression will facilitate the identification of novel biomarkers for early diagnosis and therapy of CRC, thus improving the outcome of patients with CRC. Over the past decade, miRNAs emerged as a new class of gene regulators involved in a variety of cancers $(17,18)$. 
A

HT29

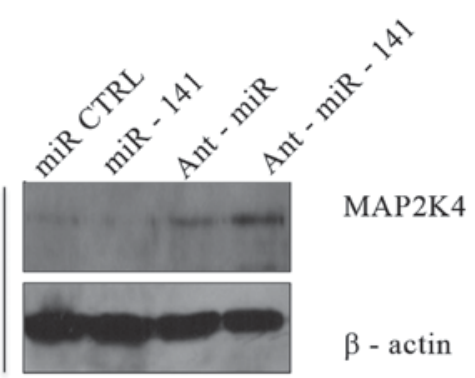

T84

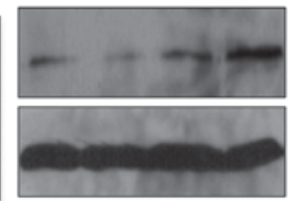

MAP2K4

$\beta$ - actin

LS174

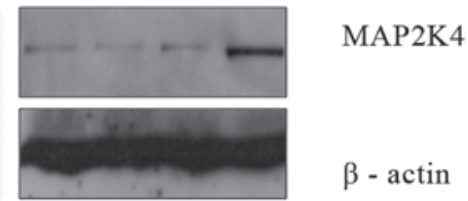

B

3' gguAGA-AAUGGUCUGUCACAAu 5' hsa-miR-141

| | | |: : | : | || || |

1233: 5' aucUCUCAUAUUCGCUAGUGUUu 3' MAP2K4 WT

5' aucUCUCAUAUUCGCUCACACCu 3' MAP2K4-Mut

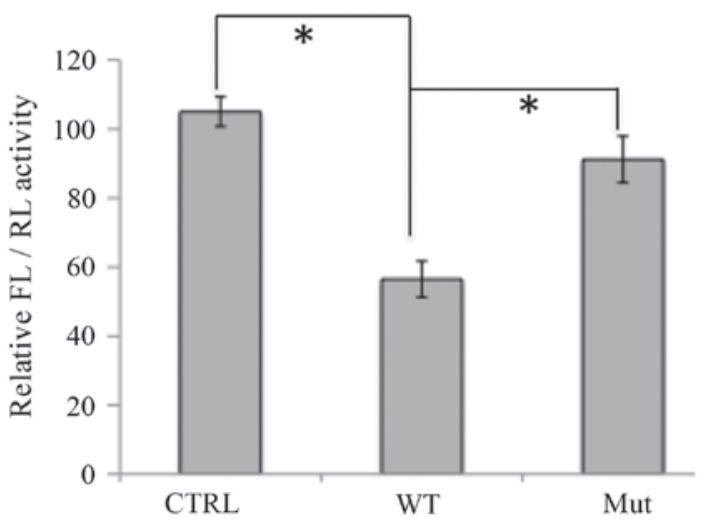

Figure 3. MAP2K4 is one of the miR-141 targets. (A) The MAP2K4 expression levels in colonic adenocarcinoma cell lines upon transfection with miR-141 mimics, Ant-miR-141 and their respective control (miR CTRL or Ant-miR, respectively; $40 \mathrm{nM}$ ) were determined by western blotting. (B) Top panel: Predicted target site of miR-141 in the 3'-UTR region of MAP2K4 messenger RNA. Bottom panel: The luciferase activity in the presence of both WT or Mut MAP2K4 3'-UTR and miR-141 mimics was compared with that of the control. "P<0.01 $(n=3)$. miR, microRNA; CTRL, control; Ant, antagomir; MAP2K4, mitogen-activated protein kinase kinase 4; UTR, untranslated region; FL, firefly luciferase; RL, Renilla luciferase; WT, wild type; Mut, mutant; hsa, Homo sapiens.

The present study has demonstrated for the first time that miR-141 plays a critical role in colonic tumorigenesis by inhibiting the tumor-suppressor gene MAK2K4 to promote cancer proliferation. Our results support that miR-141 can be used for early diagnosis and as a potential drug target in the treatment of CRC.

miRNAs are an abundant class of endogenous small RNA molecules of 20-25 nucleotides in length $(10,29)$. As important regulators in animals and plants, miRNAs regulate
A

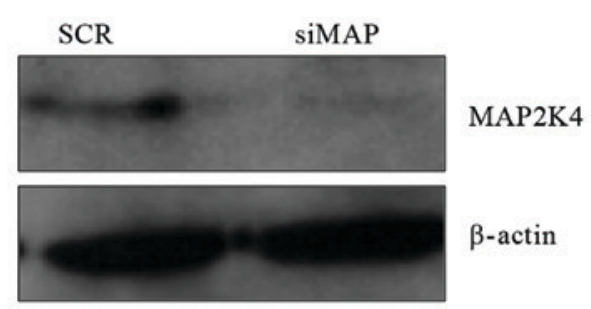

B

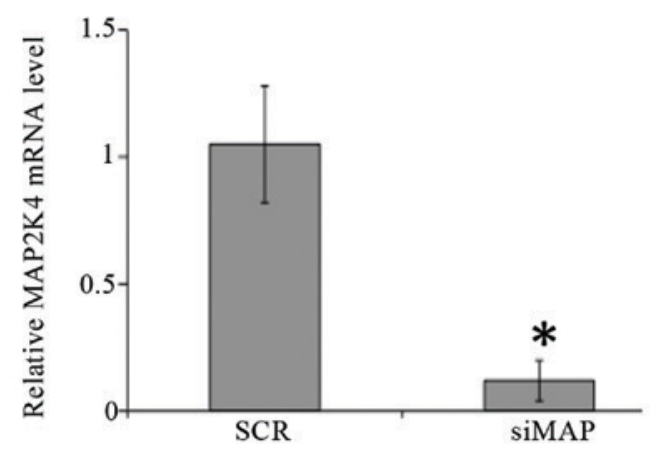

C

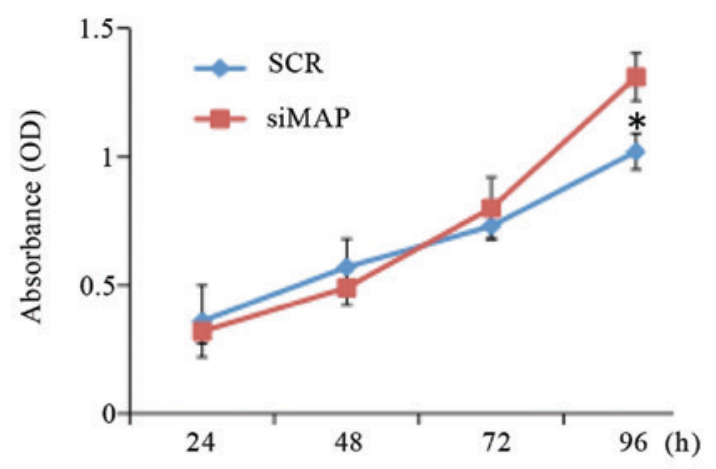

D

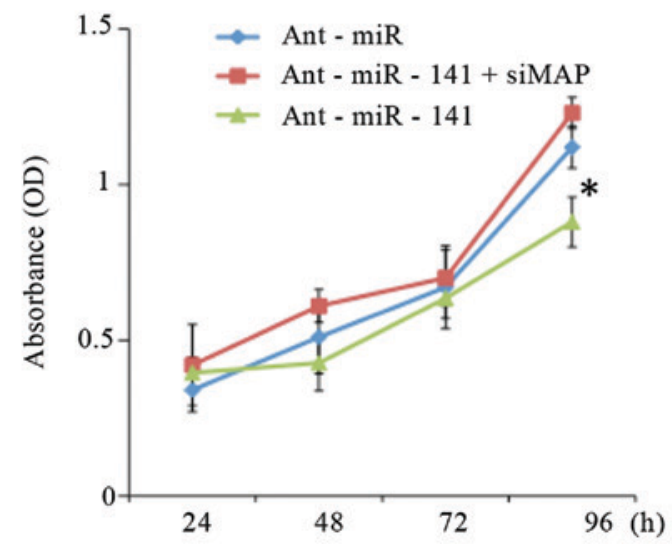

Figure 4. miR-141 promotes CAC proliferation by inhibiting MAP2K4. (A and B) The MAP2K4 levels were assessed by (A) western blotting and (B) reverse transcription-quantitative polymerase chain reaction following transfection of HT29 cells with non-targeting siRNAs (SCR) or siRNAs targeting MAP2K4 mRNA (siMAP). (C) The proliferation of HT29 CAC cells transiently transfected with siMAP or SCR was assessed using MTT assays. (D) The proliferation of HT29 CAC cells transiently transfected with Ant-miR-141, Ant-miR-141 + siMAP or control (Ant-miR) was analyzed by MTT proliferation assay. ${ }^{\mathrm{P}}<0.01(\mathrm{n}=3)$. miR, microRNA; Ant, antagomir; si, small interfering; SCR, siRNA control; MAP2K4, mitogen-activated protein kinase kinase 4; CAC, colonic adenocarcinoma; OD, optical density; mRNA, messenger RNA.

gene expression by either repressing protein translation or destabilizing their target mRNA levels (30-32). miRNAs can 
function as oncogenes or as tumor-suppressor genes (18). Numerous miRNAs are directly involved in the pathogenesis of human cancers, including lung, breast, brain, liver and colon cancer, as well as leukemia, by regulating cell proliferation and apoptosis, processes that are important in cancer formation (17). Overexpressed miRNAs in cancers, such as mir-17-92, may function as oncogenes and may promote cancer development by negatively regulating tumor-suppressor genes and/or genes that control cell differentiation or apoptosis (33). Underexpressed miRNAs in cancers, such as let-7, function as tumor-suppressor genes, and may inhibit cancers by regulating oncogenes and/or genes that control cell differentiation or apoptosis (34). miRNA expression profiles may become useful biomarkers for cancer diagnostics. In addition, miRNA therapy could be a powerful tool for cancer prevention and therapeutics.

With regard to the regulation of miR-141, multiple lines of evidence support that epigenetic mechanisms are involved in the regulation of miR-141 expression in both normal and cancer cells (35). There is an inverse correlation between the expression and DNA methylation states of miR-141 in normal human and mouse cell lines, as well as in human breast and prostate cancer cell lines $(35,36)$. In addition, different histone codes exist between miR-141 expressing and non-expressing cells that accurately represent the expression and DNA methylation states (35). Additionally, the epigenetic modifier 5-aza-2'-deoxycytidine relieves the repression of miR-141 in cancer cell lines (35). Collectively, these findings indicate that miR-141 is an evolutionarily conserved epigenetically labile miRNA cluster (35). Since de novo DNA (cytosine-5-)-methyltransferase 3 beta (DNMT3B) is frequently repressed in human CRC cell lines and primary CRC tumors by aberrant DNA hypermethylation of its distal promoter (37), we hypothesize that upregulation of miR-141 in CRC may result from a reduction in DNMT3B, and that DNMT3B is likely to be responsible for the hypermethylation of miR-141, which usually occurs in healthy colon tissues. However, this hypothesis deserves further investigation.

Our understanding of the biological functions of miRNAs relies on the identification of their specific target genes. The prediction of the majority of miRNA-target sequence matching is based on imperfect complementarity of the miRNA with the 3'-UTRs of its target mRNAs (38). The requirement for complementarity of the 5' seed region could result in hundreds of possible targets (39). In the present study, MAP2K4 was experimentally validated as a direct target of miR-141 in CRC cells, adding information to previously reported cell types (23).

Numerous studies have demonstrated that MAP2K4 is a putative tumor-suppressor gene $(24,25,40)$. By systematically characterizing the biochemical properties of a large panel of cancer-associated MAP2K4 mutations, Ahn et al examined the consequences of MAP2K4 inactivation in genetically engineered mouse models of human lung cancer driven by mutant KRAS alone or in combination with mutant tumor protein p53 (TP53), and profiled transcriptional changes induced by MAP2K4 depletion in KRAS/TP53-mutant lung adenocarcinoma cells (24). The findings from that study revealed that MAP2K4 functions as a tumor suppressor in lung adenocarcinoma and inhibits tumor cell invasion by decreasing peroxisome proliferator-activated receptor $\gamma 2$ expression (24).
In addition, MAP2K4 inactivation increased the multiplicity and accelerated the growth of incipient lung neoplasias, and promoted the invasion and metastasis of KRAS/TP53-mutant lung adenocarcinoma cells, leading to the conclusion that MAP2K4 exerts its tumor-suppressor activity at both the early and late stages of lung tumorigenesis (24). Given the inverse correlation of miR-141 and MAP2K4 in CRC identified in the present study, we speculate that the signaling pathway of miR-141-MAP2K4 is likely to be associated with the prognosis of CRC. Targeting this signaling pathway may represent a novel approach for the treatment of CRC.

In summary, the present study reports that miR-141 is frequently upregulated in CRC and is associated with cancer cell proliferation of CRC. It further demonstrates that miR-141 promotes cell growth of CRC cell by repressing the tumor-suppressor gene MAP2K4. We propose that the strategy of targeting miR-141 may provide an effective therapeutic approach for CRC patients who have exhausted other modes of treatment.

\section{References}

1. Cheng H, Zhang L, Cogdell DE, Zheng H, Schetter AJ, Nykter M, Harris CC, Chen K, Hamilton SR and Zhang W: Circulating plasma MiR-141 is a novel biomarker for metastatic colon cancer and predicts poor prognosis. PLoS One 6: e17745, 2011.

2. Pox CP: Controversies in colorectal cancer screening. Digestion 89: 274-281, 2014.

3. Zhang YL, Zhang ZS, Wu BP and Zhou DY: Early diagnosis for colorectal cancer in China. World J Gastroenterol 8: 21-25, 2002.

4. American Cancer Society: What are the survival rates for colorectal cancer by stage? http://www.cancer. org/Cancer/ColonandRectumCancer/DetailedGuide/colorectal-ca ncer-survival-rates. Accessed October 15, 2015.

5. O'Connell JB, Maggard MA and Ko CY: Colon cancer survival rates with the new American Joint Committee on Cancer sixth edition staging. J Natl Cancer Inst 96: 1420-1425, 2004.

6. Brenner H, Kloor M and Pox CP: Colorectal cancer. Lancet 383: 1490-1502, 2014

7. Bijan MD and Azadeh S: An overview of colorectal cancer survival rates and prognosis in Asia. World J Gastrointest Oncol 4: 71-75, 2012.

8. Takuji T, Mayu T, Takahiro T and Rikako I: Biomarkers for Colorectal Cancer. Int J Mol Sci 11: 3209-3225, 2010.

9. Bartel DP and Chen CZ: Micromanagers of gene expression: The potentially widespread influence of metazoan microRNAs. Nat Rev Genet 5: 396-400, 2004.

10. Bartel DP: MicroRNAs: Genomics, biogenesis, mechanism, and function. Cell 116: 281-297, 2004.

11. Jansson MD and Lund AH: MicroRNA and cancer. Mol Oncol 6: 590-610, 2012.

12. Ambros V: The functions of animal microRNAs. Nature 431: 350-355, 2004

13. Filipowicz W, Bhattacharyya SN and Sonenberg N: Mechanisms of post-transcriptional regulation by microRNAs: Are the answers in sight? Nat Rev Genet 9: 102-114, 2008.

14. Lewis BP, Burge CB and Bartel DP: Conserved seed pairing, often flanked by adenosines, indicates that thousands of human genes are microRNA targets. Cell 120: 15-20, 2005.

15. Sonja H and Damjan G: MicroRNAs as Novel Biomarkers in Colorectal Cancer. Front Genet 3: 180, 2012.

16. Lu J, Getz G, Miska EA, Alvarez-Saavedra E, Lamb J, Peck D, Sweet-Cordero A, Ebert BL, Mak RH, Ferrando AA, et al: MicroRNA expression profiles classify human cancers. Nature 435: 834-838, 2005.

17. Hwang HW and Mendell JT: MicroRNAs in cell proliferation, cell death, and tumorigenesis. Br J Cancer 94: 776-780, 2006.

18. Kent OA and Mendell JT: A small piece in the cancer puzzle: microRNAs as tumor suppressors and oncogenes. Oncogene 25: 6188-6196, 2006.

19. Iorio MV and Croce CM: MicroRNA involvement in human cancer. Carcinogenesis 33: 1126-1133, 2012. 
20. Iorio MV and Croce CM: MicroRNA dysregulation in cancer: Diagnostics, monitoring and therapeutics. A comprehensive review. EMBO Mol Med 4: 143-159, 2012.

21. Brunet Vega A, Pericay C, Moya I, Ferrer A, Dotor E, Pisa A, Casalots A, Serra-Aracil X, Oliva JC, Ruiz A and Saigí E: MicroRNA expression profile in stage III colorectal cancer: Circulating miR-18a and miR-29a as promising biomarkers Oncol Rep 30: 320-326, 2013.

22. Si ML, Zhu S, Wu H, Lu Z, Wu F and Mo YY: miR-21-mediated tumor growth. Oncogene 26: 2799-2803, 2007.

23. Zhang L, Deng T, Li X, Liu H, Zhou H, Ma J, Wu M, Zhou M, Shen S, Li X, et al: microRNA-141 is involved in a nasopharyngeal carcinoma-related genes network. Carcinogenesis 31 : 559-566, 2010

24. Ahn YH, Yang Y, Gibbons DL, Creighton CJ, Yang F, Wistuba II, Lin W, Thilaganathan N, Alvarez CA, Roybal J, et al: Map2k4 functions as a tumor suppressor in lung adenocarcinoma and inhibits tumor cell invasion by decreasing peroxisome proliferator-activated receptor $\gamma 2$ expression. Mol Cell Biol 31: 4270-4285, 2011

25. Davis SJ, Choong DY, Ramakrishna M, Ryland GL, Campbell IG and Gorringe KL: Analysis of the mitogen-activated protein kinase kinase 4 (MAP2K4) tumor suppressor gene in ovarian cancer. BMC Cancer 11: 173, 2011.

26. Livak KJ and Schmittgen TD: Analysis of relative gene expression data using real-time quantitative PCR and the 2(-Delta Delta C(T)) method. Methods 25: 402-408, 2001.

27. He D, Miao H, Xu Y, Xiong L, Wang Y, Xiang H, Zhang H and Zhang Z: MiR-371-5p facilitates pancreatic cancer cell proliferation and decreases patient survival. PLoS One 9: e112930, 2014

28. Krutzfeldt J, Rajewsky N, Braich R, Rajeev KG, Tuschl T, Manoharan M and Stoffel M: Silencing of microRNAs in vivo with 'antagomirs'. Nature 438: 685-689, 2005.

29. Ambros V: microRNAs: Tiny regulators with great potential. Cell 107: 823-826, 2001.

30. Wang B, Love TM, Call ME, Doench JG and Novina CD: Recapitulation of short RNA-directed translational gene silencing in vitro. Mol Cell 22: 553-560, 2006.
31. Wakiyama M, Takimoto K, Ohara O and Yokoyama S: Let-7 microRNA-mediated mRNA deadenylation and translational repression in a mammalian cell-free system. Genes Dev 21: $1857-1862,2007$.

32. Djuranovic S, Nahvi A and Green R: miRNA-mediated gene silencing by translational repression followed by mRNA deadenylation and decay. Science 336: 237-240, 2012.

33. Olive V, Bennett MJ, Walker JC, Ma C, Jiang I, Cordon-Cardo C, Li QJ, Lowe SW, Hannon GJ and He L: miR-19 is a key oncogenic component of mir-17-92. Genes Dev 23: 2839-2849, 2009.

34. Lee YS and Dutta A: The tumor suppressor microRNA let-7 represses the HMGA2 oncogene. Genes Dev 21: 1025-1030, 2007.

35. Vrba L, Jensen TJ, Garbe JC,Heimark RL, Cress AE, Dickinson S, Stampfer MR and Futscher BW: Role for DNA methylation in the regulation of miR-200c and miR-141 expression in normal and cancer cells. PLoS One 5: e8697, 2010.

36. Batista L, Bourachot B, Mateescu B, Reyal F and Mechta-Grigoriou F: Regulation of miR-200c/141 expression by intergenic DNA-looping and transcriptional read-through. Nat Commun 7: 8959, 2016.

37. Huidobro C, Urdinguio RG, Rodriguez RM, Mangas C, Calvanese V, Martínez-Camblor P, Ferrero C, Parra-Blanco A, Rodrigo L, Obaya AJ, et al: A DNA methylation signature associated with aberrant promoter DNA hypermethylation of DNMT3B in human colorectal cancer. Eur J Cancer 48: 2270-2281, 2012.

38. Felekkis K, Touvana E, Stefanou Ch and Deltas C: microRNAs: A newly described class of encoded molecules that play a role in health and disease. Hippokratia 14: 236-240, 2010.

39. Hibio N, Hino K, Shimizu E, Nagata Y and Ui-Tei K: Stability of miRNA 5'terminal and seed regions is correlated with experimentally observed miRNA-mediated silencing efficacy. Sci Rep 2: 996, 2012.

40. Teng DH, Perry WL III, Hogan JK, Baumgard M, Bell R, Berry S, Davis T, Frank D, Frye C, Hattier T, et al: Human mitogen-activated protein kinase kinase 4 as a candidate tumor suppressor. Cancer Res 57: 4177-4182, 1997. 